\title{
The Integration of Internal and External Training Load Metrics in Hurling
}

\author{
by \\ Shane Malone ${ }^{1,2}$, Dominic Doran ${ }^{2}$, Ibrahim Akubat ${ }^{3}$, Kieran Collins $^{1}$
}

The current study aimed to assess the relationship between the hurling player's fitness profile and integrated training load (TL) metrics. Twenty-five hurling players performed treadmill testing for VO2max, the speed at blood lactate concentrations of $2 \mathrm{mmol} \bullet \mathrm{L}-1(v L T)$ and $4 \mathrm{mmol} \bullet \mathrm{L}-1(v O B L A)$ and the heart rate-blood lactate profile for calculation of individual training impulse (iTRIMP). The total distance (TD; $m)$, high speed distance (HSD; $m)$ and sprint distance $(S D ; m)$ covered were measured using GPS technology (4-Hz, VX Sport, Lower Hutt, New Zealand) which allowed for the measurement of the external TL. The external TL was divided by the internal TL to form integration ratios. Pearson correlation analyses allowed for the assessment of the relationships between fitness measures and the ratios to performance during simulated match play. External measures of the TL alone showed limited correlations with fitness measures. Integrated TL ratios showed significant relationships with fitness measures in players. TD:iTRIMP was correlated with aerobic fitness measures VO2max ( $r=0.524 ; p=0.006 ; 95 \% \mathrm{CI}: 0.224$ to 0.754 ; large) and $v O B L A(r=0.559 ; p=0.003 ; 95 \%$ CI: 0.254 to 0.854; large). HSD:iTRIMP also correlated with aerobic markers for fitness $v L T$ ( $r=0.502 ; p=0.009 ; 95 \%$ CI: 0.204 to 0.801; large); vOBLA ( $r=0.407 ; p=0.039 ; 95 \%$ CI: 0.024 to 0.644; moderate). Interestingly SD:iTRIMP also showed significant correlations with $v L T(r=0.611 ; p=$ 0.001; 95\% CI: 0.324 to 0.754; large). The current study showed that TL ratios can provide practitioners with a measure of fitness as external performance alone showed limited relationships with aerobic fitness measures.

Key words: ITRIMP; intermittent exercise; lactate; heart rate; training load ratios.

\section{Introduction}

Hurling is a stick and ball invasion field sport native to Ireland (Reilly and Collins, 2008). The movement profile of players is stochastic in nature, where the timing of high speed running follows the ebb and flow of competitive play (Reilly and Collins, 2008). Despite its spectator popularity very few research investigations have been conducted on hurling cohorts (Reilly and Collins, 2008). The training-recovery relationship is an important consideration in the training prescription of players and a clear understanding of training loads can help inform practice. Recent reports have linked the training load and inadequate fitness to the high number of injuries observed within the Gaelic sports (Blake et al., 2014). Specifically Blake et al. (2014) reported that the injury incidence rate for match play $(61.75 / 1000 \mathrm{hr})$ is 21 times that of training $(2.99 / 1000 \mathrm{hr})$. This suggests that training does not adequately prepare players for the competitive demands of match-play.

The training load (TL) in field sports can be divided into internal (heart rate, RPE) or external (distance covered) responses. An

\footnotetext{
1 - Gaelic Sport Research Centre, Institute of Technology Tallaght Dublin, Tallaght, Dublin, Ireland.

2 - Research Institute for Sport and Exercise Sciences, The Tom Reilly Building, Liverpool John Moores University, Henry Cotton Campus, Webster Street, Liverpool, UK.

3 - Department of Physical Education and Sports Studies, Newman University, Birmingham, UK.
} 
understanding of the TL allows for a more individualised understanding of the doseresponse relationship of training and match play (Impellizzeri et al., 2005; Stagano et al., 2007). Recent advancement in technology has improved the ease to which locomotion data collection can take place. There has been an increase in monitoring of the external training load in match and training situations through the use of global positioning satellite systems (GPS) (Casamichana et al., 2014, 2015; Evans et al., 2015; Malone et al., 2015). The advent of this technology has resulted in total distance covered and distance covered at arbitrary demarcated thresholds of high speed locomotion being utilised to quantify the TL. The poor sensitivity and variability of these performance metrics within match play and training environments has been previously highlighted (Gregson et al., 2010; Kempton et al., 2014; McLaren et al., 2015; Stevens et al., 2016). As a consequence these metrics may be unreliable to gain full appreciation of the TL.

The understanding of the dose-response relationship has been advocated as an important consideration for coaches within the training process. Monitoring the TL in team sports is difficult due to different exercise designs resulting in different physiological and mechanical demands. Additional complications are presented in the form of inter-individual responses to the prescribed exercise (Akubat et al., 2012). Given that the HR-O2 consumption relationship appears to be valid even during intermittent exercise (Esposito et al., 2004), the iTRIMP methodology has potential for use for monitoring the doseresponse of players to specific training programs within a team sport environment (Manzi et al., 2013).

Indeed, measures of the internal TL have been validated with a dose-response relationship in team sports (Akubat et al., 2012; Manzi et al., 2013). Both studies demonstrated that the fully individualized training impulse (iTRIMP) showed relationships with changes in aerobic-fitness measures of soccer players. Previous studies (Manzi et al., 2013) have shown that iTRIMP measures over a training period were correlated to the percentage improvement in $\mathrm{VO} 2 \mathrm{max}(\mathrm{r}=$ 0.77, $\mathrm{p}=0.002)$ and Yo-YoIR1 performance $(\mathrm{r}=$ $0.69, \mathrm{p}=0.009)$. Further advancement of this measure was proposed by Akubat et al. (2014) through the integration of external (total distance [TD]; high speed distance [HSD]) and internal loading parameters (iTRIMP). Interestingly, these measures have shown a relationship with aerobic fitness measures in soccer players (Akubat et al., 2014). The investigation reported that both TD:iTRIMP showed moderate to large correlations with vOBLA $(\mathrm{r}=0.65 ; \mathrm{p}=004$; large $)$ and vOBLA $(r=0.69 ; p=0.03$; large $)$, with TD not significantly related to $\mathrm{vLT}(\mathrm{r}=0.28 ; \mathrm{p}=0.43$; small) and vOBLA ( $r=0.14 ; \mathrm{p}=0.69$; small). This highlights the potential merits of using TL ratios to better quantify the dose-response of the TL. Furthermore, studies within the rugby league (Weaving et al., 2014) have shown that the integration of TL measures better explain a greater proportion of training component variance that internal or external measures of load alone fail to fully explain.

At elite levels of hurling a disproportionate number of injuries occur during match play (Murphy et al., 2007) with most occurring in the last quarter of games due to increased fatigue states (Blake et al., 2014). This is in line with previous investigations (Watson, 1996) that have reported injury occurrences in hurling populations which are attributable to poor conditioning of players. Given such a scenario, it would appear that a situation where an analysis and assessment of aerobic fitness training through data collected routinely at elite levels could prove useful. Considering the potential ease of quantifying the HR (internal load) and the GPS (external load), both the internal and external output of exercise can be measured. If external:internal measures relate to measures of fitness that predetermine a given performance, more frequent analysis of training status and match performance could be made by practitioners. This would allow for more informed and proactive decisions related to player's fitness and fatigue status, therefore, providing a more thorough understanding of the training load paradigm in intermittent team sports. Hence, the current study investigated whether information such as the HR and distance could potentially be used to assess the match play load. Therefore, the study aimed to examine a novel approach of integrating external and internal workload variables as a ratio during hurling specific simulated match play. Finally, the study aimed to 
quantify the relationship of integrated TL ratios and aerobic fitness measures in hurling players.

\section{Material and Methods}

\section{Participants}

Twenty-five hurling players (age: $24 \pm 4$ yrs; body height: $1.80 \pm 0.02 \mathrm{~m}$; body mass: $78 \pm 3 \mathrm{~kg}$; $\mathrm{VO}_{2 \max }: 57.78 \pm 3.05 \mathrm{~mL} \cdot \mathrm{kg}^{-1} \cdot \mathrm{min}^{-1}$ ) took part in this study. All subjects provided written informed consent to the experimental procedures after all possible benefits and risks were explained to them. Ethical consent for the study was granted by the research ethics committee of the Institute of technology Tallaght. The study conformed to the Codes of Ethics of the World Medical Association (Declaration of Helsinki, 1964)

\section{Procedures}

The study was completed over a 3 week period. During the $1^{\text {st }}$ week an incremental $\mathrm{VO}_{2 \max }$ and lactate threshold test $\left(\mathrm{VO}_{2} / \mathrm{LT}\right)$ was conducted. The $\mathrm{VO}_{2} / \mathrm{LT}$ consisted of five $\left(8,10,12,14 \& 16 \mathrm{~km} \cdot \mathrm{h}^{-1}\right)$ 4 min stages followed by an increase in speed at a rate of $0.2 \mathrm{~km} \cdot \mathrm{h}^{-1}$ every $12 \mathrm{~s}$ until exhaustion was reached. The treadmill gradient was set at $1 \%$ for the entire test to reflect the energetic cost of outdoor running (Jones and Doust, 1996). During the incremental test, each stage was separated by a $1 \mathrm{~min}$ recovery period where capillary blood lactate was taken (Lactate Plus, Nova Medical) in duplicate, with the mean value for each stage recorded. The velocity at $2 \mathrm{mmol} \cdot \mathrm{L}^{-1}(\mathrm{vLT})$ and velocity at $4 \mathrm{mmol} \cdot \mathrm{L}^{-1}$ (vOBLA) were considered as markers of aerobic fitness. Maximal aerobic capacity $\left(\mathrm{VO}_{2 \max }\right)$ was assessed during the $\mathrm{VO}_{2} / \mathrm{LT}$ through breath by breath analysis (Cosmed $\mathrm{K}^{2} \mathrm{~b}^{2}$, Rome, Italy). The system was calibrated before each test according to the manufacturer's instructions. $\mathrm{VO}_{2 \max }$ was recorded as the highest mean $\mathrm{VO}_{2}$ obtained for a $1 \mathrm{~min}$ period with the following criterion met: (1) a plateau in $\mathrm{VO}_{2}$ despite increasing treadmill speed (2) a respiratory ratio above 1.10 (3) attainment of the age predicted HR (Lucia et al., 2004). The HR was recorded using HR belts (Polar Team System, Polar Electro, OY, Finland).

The mean HR during the last minute of each stage was utilised to ascertain the individualized HRblood lactate relationship for the calculation of iTRIMP (AU) weightings. Finally, the peak HR during the incremental treadmill test was considered the peak HR of the participant. During weeks 2 and 3 and at least 5 days after the $\mathrm{VO}_{2 \max } / \mathrm{LT}$ test, participants completed a hurling match play simulation protocol. The hurling simulation protocol was chosen given its accurate physiological replication of hurling match play (Collins et al., 2010). Before conducting the study, test retest reliability analysis was conducted on the hurling match play simulation protocol to ensure that the test provides external validity and reliability. Results showed that there was no significant difference between the $1^{\text {st }}$ and $2^{\text {nd }}$ trial of the hurling simulation protocol $(7411 \pm 354$ $\mathrm{m} ; 7311 \pm 362 \mathrm{~m} ; p=0.19$; trivial). The coefficient of variation $(\mathrm{CV})$ was $1.9 \%$ (95\% CI: 1.1-4.8) highlighting the reliability of the simulation.

The hurling match play simulation protocol was completed at the same time of the day (10.30 $14.00 \mathrm{hr}$ ) to account for any circadian variation in performance. During the simulation participants wore an individual GPS unit (VXsport, Lower Hutt, New Zealand, Issue: 330a, Firmware: 3.26.7.0) sampling at $4 \mathrm{~Hz}$ and containing a triaxial acceloremter and a magnetometer in a total of 30 games. The GPS unit (mass: $76 \mathrm{~g}$; $48 \mathrm{~mm}$ x $20 \mathrm{~mm} \times 87$ $\mathrm{mm}$ ) was encased within a protective harness between the player's shoulder blades in the upper thoracic-spine region what ensured that players' range of movement in the upper limbs and torso was not restricted. The system had been validated, deemed accurate and reliable during intermittent exercise (Buchheit et al., 2014). Following each match GPS data were downloaded using the same proprietary software (VXSport View, New Zealand). Each file was trimmed so that only data recorded when the player was on the field was included for further analysis. The proprietary software provided instantaneous raw velocity data at $0.25 \mathrm{~s}$ intervals, which were then exported and placed into a customised Microsoft Excel spreadsheet (Microsoft, Redmond, USA). The spreadsheet allowed analysis of distance covered $(\mathrm{m})$ in the following categories: total distance (TD), high speed distance (HSD: $\geq 17 \mathrm{~km} \cdot \mathrm{h}^{-1}$ ) and sprint distance (SD: $\geq 22 \mathrm{~km} \cdot \mathrm{h}^{-1}$ ). Internal loading was measured as individualised training impulses (iTRIMP) (Manzi et al., 2013).

\section{Statistical Analysis}

Results are reported as mean \pm SD. Descriptive analysis and assumptions of normality were verified prior to parametric statistical analysis. Pearson correlation coefficients were used to assess the relationships between (1) aerobic fitness and performance, and (2) distance:iTRIMP and fitness. Qualitative interpretations of the correlation coefficients and 
the $95 \%$ confidence intervals $(\mathrm{CI})$ as defined by Hopkins (2004) (0-.09 trivial, .10-.29 small, .30-.49 moderate, . $.50-.69$ large, $.70-.89$ very large, $.90-.99$ nearly perfect, 1 perfect) are provided for all correlations. All statistical analyses were performed using SPSS for Windows (Version 22, SPSS Inc. Chicago, IL, USA) with statistical significance set at an accepted level of $p<0.05$.

\section{Results}

The TD, HSD and SD covered was $7604 \pm 510$ $\mathrm{m}, 1623 \pm 149 \mathrm{~m}$ and $300 \pm 47 \mathrm{~m}$, respectively. The internal TL (iTRIMP) of simulated match play was $534 \pm 74$ AU. The relationships between external load measures and fitness are shown in Table 1. Specifically, TD covered during the match simulation was not significantly correlated to aerobic fitness measures i.e. vLT $(\mathrm{r}=-0.066 ; p=$ 0.747; $95 \%$ CI: 0.014 to 0.081 ; trivial), $\mathrm{VO}_{2 \max }(\mathrm{r}=$ $0.152 ; p=0.458 ; 95 \%$ CI: 0.114 to 0.261 ; small) and vOBLA ( $\mathrm{r}=0.014 ; p=0.945 ; 95 \% \mathrm{CI}:-0.014$ to 0.084; trivial). Interestingly, similar trends were observed in relation to HSD and aerobic fitness measures. HSD was not related to players $\mathrm{VO}_{2 \max }$ ( $\mathrm{r}=0.086 ; p=0.677 ; 95 \%$ CI: 0.014 to 0.092 ; trivial $)$ and vOBLA ( $\mathrm{r}=0.076 ; p=0.712 ; 95 \%$ CI: 0.034 to
0.090; trivial). In contrast to previous observations, HSD was significantly related to $\operatorname{vLT}(\mathrm{r}=0.485 ; p=0.012 ; 95 \%$ CI: 0.314 to 0.619 ; moderate). Finally, SD showed no significant correlation with vLT $(r=-0.189 ; p=0.356 ; 95 \%$ CI: 0.014 to 0.091 ; trivial), $\mathrm{VO}_{2 \max }(\mathrm{r}=0.094 ; p=0.647$; 95\% CI: 0.074 to 0.102 ; trivial) and vOBLA ( $\mathrm{r}=$ $0.012 ; p=0.955 ; 95 \%$ CI: 0.004 to 0.074 ; trivial). The integration of TL measures was shown to provide significant correlations with aerobic fitness measures. The relationship between TL integration ratios and aerobic fitness measures is shown in Table 2. Specifically as shown in Figure 1, TD:iTRIMP showed significant correlations with $\mathrm{VO}_{2 \max }(\mathrm{r}=0.524 ; p=0.006 ; 95 \% \mathrm{CI}: 0.224$ to 0.754 ; large) and vOBLA ( $\mathrm{r}=0.559 ; p=0.003 ; 95 \%$ CI: 0.314 to 0.774 ; large). Similar trends were shown in Figure 2 for HSD:iTRIMP and aerobic markers for fitness measures. HSD:iTRIMP was significantly related to vLT $(\mathrm{r}=0.502 ; p=0.009$; 95\% CI: 0.214 to 0.861 ; large) and vOBLA ( $\mathrm{r}=$ $0.407 ; p=0.039 ; 95 \%$ CI: 0.204 to 0.641 ; moderate). Finally, the integration of SD:iTRIMP shown in Figure 3 was significantly related to $\mathrm{vLT}(\mathrm{r}=$ $0.611 ; p=0.001 ; 95 \%$ CI: 0.414 to 0.841 ; large).

\section{Table 1}

The correlation of the external TL with measures of aerobic fitness

External TL $\quad \operatorname{VO}_{2 \max }\left(\mathrm{mL} \cdot \mathrm{kg}^{-1} \cdot \mathrm{min}^{-1}\right) \quad \mathrm{vLT}\left(\mathrm{km} \cdot \mathrm{h}^{-1}\right) \quad$ vOBLA $\left(\mathrm{km} \cdot \mathrm{h}^{-1}\right)$

\begin{tabular}{cccc}
\hline Total Distance (m) & -0.158 & -0.066 & 0.014 \\
High Speed Distance (m) & 0.086 & $0.485^{* *}$ & 0.076 \\
Sprint Distance (m) & 0.094 & -0.189 & 0.012 \\
\hline
\end{tabular}

High speed distance $\left(m ; \geq 17 \mathrm{~km} \cdot \mathrm{h}^{-1}\right)$; Sprint distance $\left(\mathrm{m} ; \geq 22 \mathrm{~km} \cdot \mathrm{h}^{-1}\right)$; $v L T=$ velocity at the lactate threshold; $v O B L A=$ velocity at onset of blood lactate accumulation ** Correlation is significant $p=0.012$ 

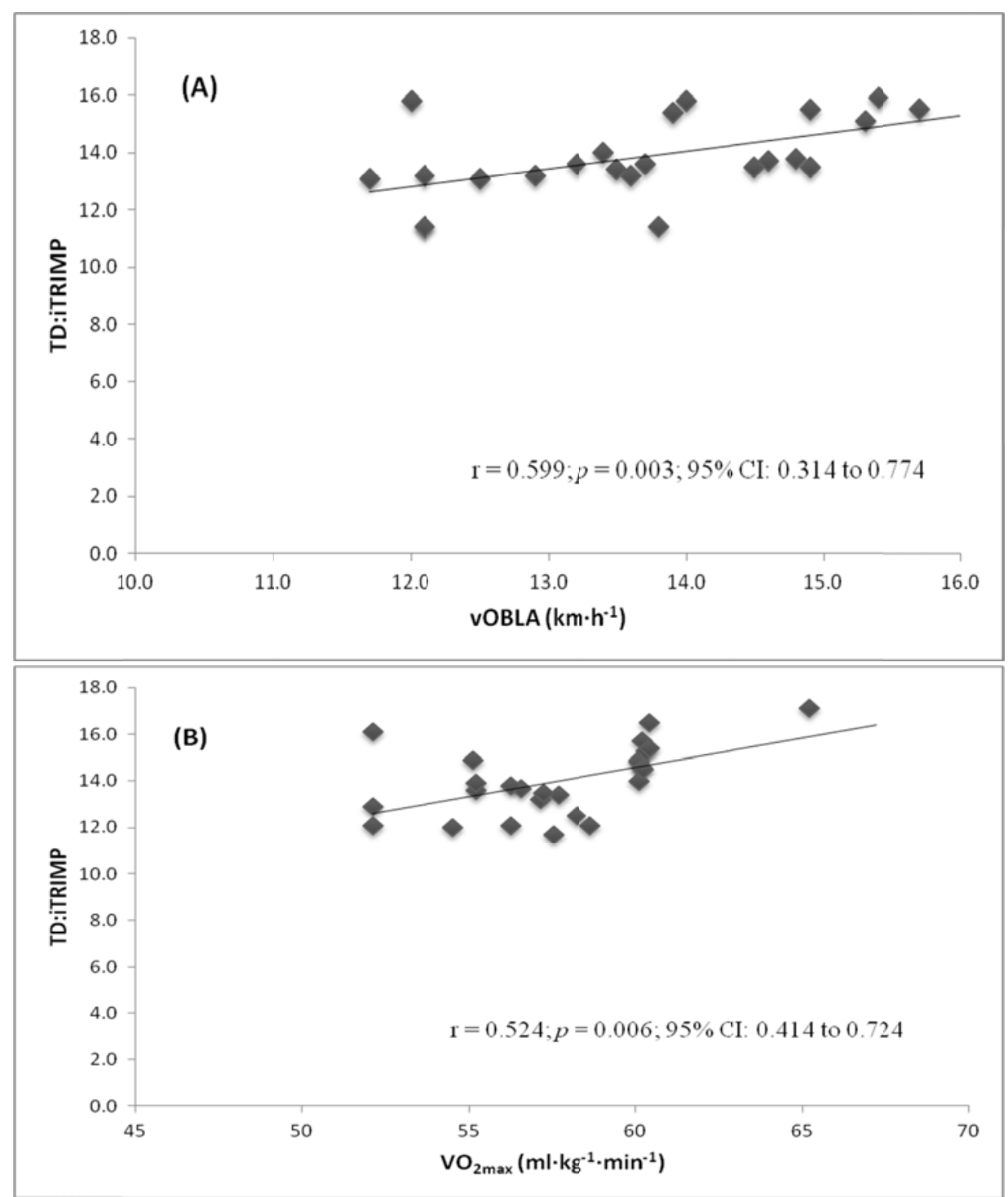

Figure 1

A scatter plot showing the relationship between (A) TD:iTRIMP and vOBLA $\left(\mathrm{km} \cdot \mathrm{h}^{-1}\right)$ (B) TD:iTRIMP and $V_{2 \max }\left(\mathrm{mL} \cdot \mathrm{kg}^{-1} \cdot \mathrm{min}^{-1}\right)$ 


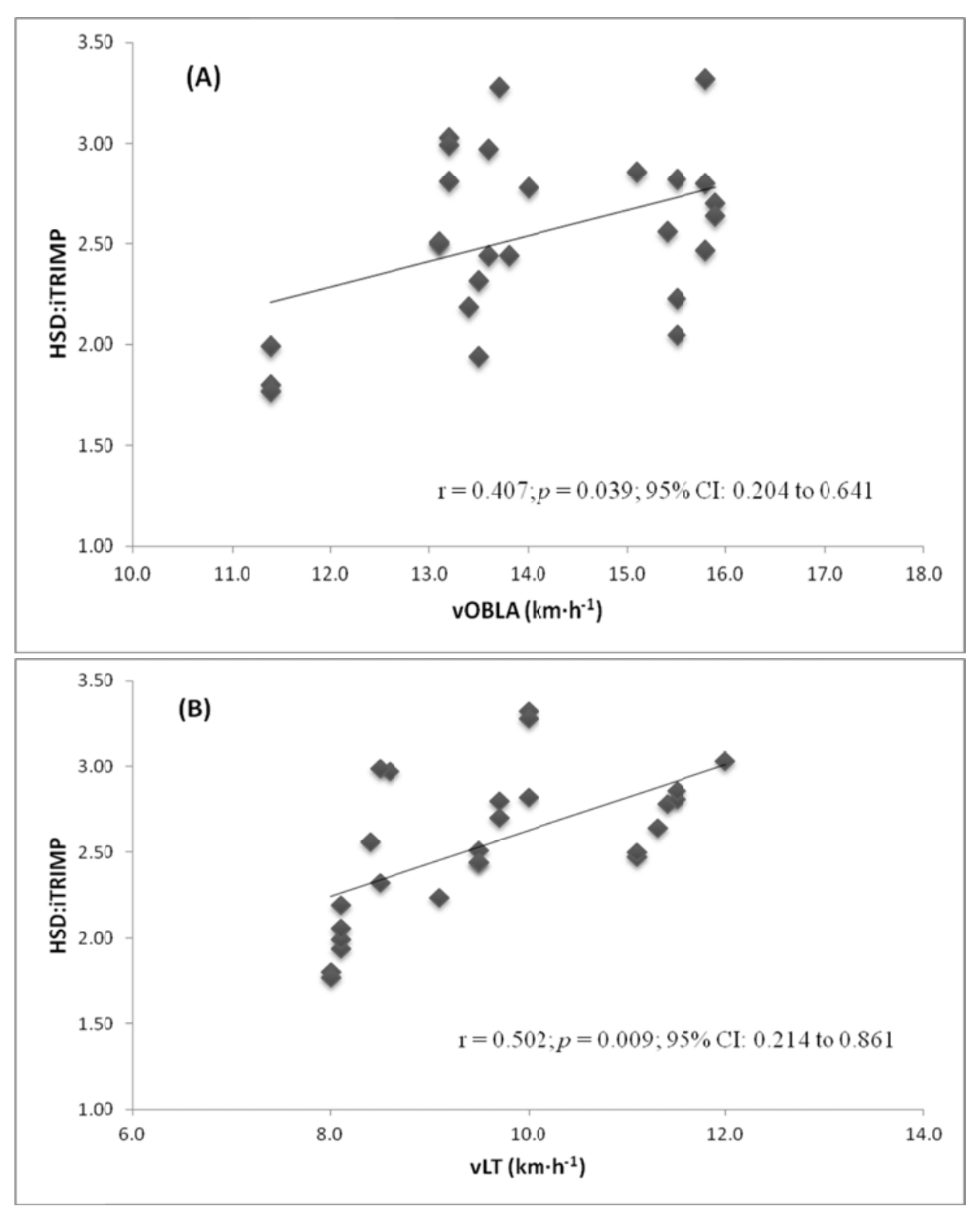

Figure 2

A scatter plot showing the relationship between (A) HSD:iTRIMP and vOBLA $\left(\mathrm{km} \cdot \mathrm{h}^{-1}\right)(B)$ HSD:iTRIMP and $v L T\left(\mathrm{~km} \cdot \mathrm{h}^{-1}\right)$ 
Table 2

The correlation of external:Internal TL ratios with measures of aerobic fitness

\begin{tabular}{lccc}
\hline $\begin{array}{l}\text { External:Internal TL } \\
\text { Ratio }\end{array}$ & $\mathrm{VO}_{2 \max }\left(\mathrm{mL} \cdot \mathrm{kg}^{-1} \cdot \mathrm{min}^{-1}\right)$ & $\begin{array}{c}\mathrm{vLT}(\mathrm{km} \cdot \mathrm{h}- \\
1)\end{array}$ & $\mathrm{vOBLA}\left(\mathrm{km} \cdot \mathrm{h}^{-1}\right)$ \\
\hline TD: iTRIMP & $0.524^{* *}$ & 0.330 & $0.599^{* *}$ \\
HSD: iTRIMP & 0.325 & $0.502^{* *}$ & $0.407^{* *}$ \\
SPRINT: iTRIMP & 0.358 & $0.611^{* *}$ & 0.170 \\
\hline
\end{tabular}

TD:iTRIMP = Total distance $(m): i T R I M P(A U) ; H S D: i T R I M P=$ High speed distance $(m)$ : iTRIMP (AU); SPRINT:iTRIMP = Sprint distance $(m): i T R I M P(A U)$; $v L T=$ velocity at the lactate threshold;

$v O B L A=$ velocity at onset of blood lactate accumulation ${ }^{* *}$ Correlation is significant $p \leq 0.05$

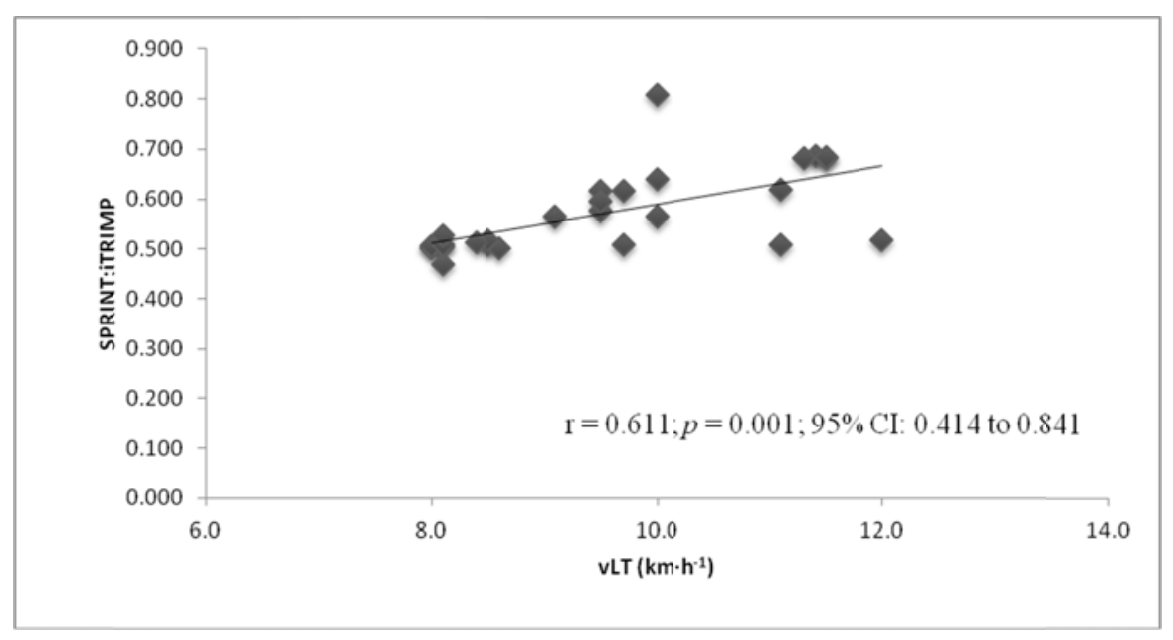

Figure 3

A scatter plot showing the relationship between SD:iTRIMP and $v L T\left(\mathrm{~km} \cdot \mathrm{h}^{-1}\right)$ 


\section{Discussion}

The current study investigated a novel approach of integrating external and internal workload variables as a ratio during hurling specific simulated match play. The results of the study show that measures of match running performance relate poorly to aerobic fitness measures. Interestingly, there appears to be a relationship between markers of aerobic fitness and high speed running performance. The relationship has been previously reported in soccer populations (Akubat et al., 2014), but this is the first study to report this relationship in a hurling population. Caution should be taken when utilising high speed running as a marker of performance, the training load and fatigue, given the match to match variability in this performance measure. Gregson and colleagues (2010) reported that high speed running performance could vary by as much as $16 \%$ (CV: $16.2 \pm 6.4 \%$, 95\% CI: 15.6$16.7 \%$ ) between competitive games. Furthermore, McLaren et al. (2015) observed large betweenmatch (within-player) variation for high speed running distance $(27.6 \% ; \pm 90 \%$ confidence limits $6.9 \%$ [forwards], $20.1 \% ; \pm 4.1 \%$ [backs]), very high speed running distance $(68 \% ; \pm 19 \%, 34.1 \% ; \pm$ $7.5 \%$ ) within rugby union. These findings question the reliability of these measures as accurate determinants of a player training load.

The non-significant relationship between external load measures and measures of fitness seen in this study is in contrast to many studies that have analysed performance in other sports such as endurance running where the relationships between similar aerobic fitness variables in endurance events show very large to nearly perfect relationships (McLaughlin et al., 2010). Possible explanations of the non-significant relationships between the external measure of performance and fitness measures seen in this study can be explained by the simulated nature of match play. During competitive hurling match play greater effort may be required at a given time due to external stimuli such as standard of opposition, tactical formation or contextual factors (Paul et al., 2015). Match simulations such as the hurling match play simulation protocol have been previously questioned as they allow players to pace themselves at levels that fail to replicate match play accurately. This pacing strategy can be related to the absence of opposition, tackling, tactical responsibility and game situation, all of which impact the locomotor performance and result in variability that may influence performance (Akubat et al., 2014). The impact of the above may explain the limited relationships between running performance and aerobic fitness variables observed during the current investigation.

With the known limitations of analysing running performance, it may be more valuable to integrate both the external (distance) and internal (iTRIMP) load to provide a ratio that considers the internal load of exercise and the external output (performance), thus providing a value representative of match day performance and fatigue (Akubat et al., 2014). Previous studies have shown the value of the basic arbitrary iTRIMP unit as a marker of assessing adaptations in fitness throughout a pre-season period within professional soccer populations (Manzi et al., 2013). Adding to this the integration method has been shown to have moderate to large correlations with aerobic fitness markers in a soccer population (Akubat et al., 2014). However, this study is the first to begin the process of integrating TL values in hurling. During the analysis TD, HSD and SD were divided by iTRIMP (AU). These values were significantly correlated to markers of aerobic fitness for hurling players. Athletes are provided with a valuable training and match day marker of the TL given Banister's (1991) proposal that performance at a given time point is a result of fitness of an individual minus the accrued fatigue. The ratio provides the practitioner with a measure of the accrued fatigue at a given time point (distance covered during the match or training session) and the fitness measures (iTRIMP). In addition, the temporal pattern of performance decrement has often been attributed to fatigue (Paul et al., 2015), therefore, this measure appears to have merits as a TL marker considering that it accounts for performance decrement and provides integration of this decrement with fitness measures.

Given the amateur nature of hurling players, frequent laboratory testing of fitness may be difficult. In addition, considering the known match to match variability in traditional external load measures such as TD and HSD, the TL integration method may provide advancement in 
the assessment of the fitness-fatigue paradigm proposed by Banister (1975). If these measures of fitness predetermine a given performance and are reduced due to fatigue, it is reasonable to expect a performance decline. Black and Dobson (2011) showed that exercise induced fatigue reduced VO2peak and the ventilatory threshold showing a temporary reduction in measures of aerobic fitness. Future research should be conducted during actual match play situations to assess if the integration ratio relationships hold true. Moreover, there is a need for the assessment of these ratios as a marker of fatigue. By determining this, we can assess the validity of this ratio as a marker of the dose-response relationship as well as allow practitioners to become more proactive and manipulate the dose rather than reacting to the response.

\section{Conclusions}

The current study presents additional findings to support the utilisation of integrated TL ratios in intermittent team sport environments. The findings suggest that the use of TL ratios provides data to practitioners that are correlated to aerobic fitness measures. In the current investigation, TD was not significantly correlated to fitness measures i.e. $\mathrm{vLT}(\mathrm{r}=-0.066 ; \mathrm{p}=0.747$;
95\% CI: 0.014 to 0.081 ; trivial), $\mathrm{VO} 2 \mathrm{max}(\mathrm{r}=0.152$; $p=0.458 ; 95 \%$ CI: 0.114 to 0.261 ; small $)$ and vOBLA $(\mathrm{r}=0.014 ; \mathrm{p}=0.945 ; 95 \% \mathrm{CI}:-0.014$ to 0.084; trivial). Similar trends were seen with HSD with this external load measure not correlated to VO2max ( $\mathrm{r}=0.086 ; \mathrm{p}=0.677 ; 95 \% \mathrm{CI}: 0.014$ to 0.092; trivial) and vOBLA $(r=0.076 ; \mathrm{p}=0.712 ; 95 \%$ CI: 0.034 to 0.090 ; trivial). Interestingly, $\mathrm{TL}$ integration ratios were correlated to fitness measures more closely than external load measures. TD:iTRIMP correlated with aerobic fitness measures i.e. $\mathrm{VO} 2 \max (r=0.524 ; \mathrm{p}=0.006$; $95 \%$ CI: 0.414 to 0.724 ; large) and vOBLA ( $\mathrm{r}=$ $0.559 ; \mathrm{p}=0.003 ; 95 \% \mathrm{CI}: 0.314$ to 0.774 ; large). Similar trends were shown for HSD:iTRIMP and aerobic markers for fitness measures i.e. $\mathrm{vLT}(\mathrm{r}=$ $0.502 ; \mathrm{p}=0.009 ; 95 \% \mathrm{CI}: 0.214$ to $0.861 ;$ large) and $\operatorname{vOBLA}(\mathrm{r}=0.407 ; \mathrm{p}=0.039 ; 95 \%$ CI: 0.204 to 0.641 ; moderate). The SD:iTRIMP showed significant correlations with vLT $(\mathrm{r}=0.611 ; \mathrm{p}=0.001 ; 95 \% \mathrm{CI}$ : 0.414 to 0.841 ; large). Overall, these measures support the use of integrated ratios as a method of monitoring training loads. Integrated ratio measures were correlated to fitness measures, as such the use of these ratios presents advancement from the use of external load measures alone in the assessment of aerobic fitness of players.

\section{Acknowledgements}

The authors would like to state that they have no conflicts of interest to declare while also thanking all the participants who committed to the extensive testing involved in this study and to all the undergraduates who helped out during the process.

\section{References}

Akubat I, Patel E, Barrett S, Abt G. Methods of monitoring the training and match load and their relationship to changes in fitness in professional youth soccer players. J Sports Sci, 2012; 30(14): 1473-1480

Akubat I, Barrett S, Abt G. Integrating the internal and external training loads in soccer. Int J Sports Physiol Perform, 2014; 9(3): 457-462

Bangsbo J, Mohr M, Krustrup P. Physical and metabolic demands of training and match-play in the elite football player. J Sports Sci, 2006; 24(7): 665-674

Banister EW, Calvert TW, Savage MV, Bach T. A system model of training for athletic performance. Aust J Sports Med, 1975; 7: 51-61

Banister EW. Modelling elite athletic performance. In: MacDougall JD, Wenger HA, and Green HJ, eds. Physiological Testing of Elite Athletes. Champaign, IL: Human Kinetics; 1991

Black CD, Dobson RM. Prior Eccentric Exercise Reduces $\mathrm{VO}_{2}$ peak and ventilatory threshold, but does not alter movement economy during cycling exercise. J Strength Cond Res, 2011; 26(9): 2530-2537

Blake C, O'Malley E, Gissane C, Murphy JC. Epidemiology of injuries in hurling: a prospective study 2007-2011. 
BMJ Open, 2014; 19(4)6: 1-7

Bradley PS, Carling C, Gomez-Diaz A, Hood P, Barnes C, Ade J, Boody M, Krustrup P, Mohr M. Match performance and physical capacities in the top three competitive standards of English professional soccer. Hum Mov Sci 2013; 32(4): 808-821

Buchheit M, Allen A, Poon TK, Mondonutti M, Gregson W, Di Salvo V. Integrating different tracking systems in football: multiple camera semi-automatic system, local positioning measurement and GPS technologies. J Sports Sci, 2014; 32(20): 1844-1857

Casamichana D, Suarez-Arrones L, Castellano J, San Roman-Quintana J. Effecr of number of touches and exercise duration on kinematic profile and heart rate response during small-sided games in soccer. J Hum Kinet, 2014; 41: 113-123

Casamichana D, San Roman-Quintana J, Casellano J, Calleja-Gonzalez J. Influence of the type of marking and the number of players on physiological and physical demands during sided games in soccer. J Hum Kinet, 2015; 47: 259-268

Collins K, Doran D, Reilly T. The physiological response to hurling match-play. In Anderson, M. eds Contemporary Ergonomics and Human Factors, 591-595; 2010

Esposito F, Impellizzeri FM, Margonato V, Vanni R, Pizzini G, Veicsteinas A. Validity of heart rate as an indicator of aerobic demand during soccer activities in amateur soccer players. Eur J App Physiol, 2004; 93: 167-172

Evans SD, Brewer C, Haigh JD, Lake, M Morton JP, Close GL. The physical demands of Super League rugby: Experiences of newly promoted franchise. Euro J Sport Sci, 2015; 15(6): 505 - 513

Gregson W, Drust B, Atkinson G, Di Salvo V. Match- to- match variability of high-speed activities in Premier League soccer. Int J Sports Med, 2010; 31(4):237-242

Hopkins WG. A New View of Statistics, 2004, available at: http:/www.sportsci.org/resource/stats/index.html; accessed on 05.01 .2015

Impellizzeri FM, Rampinini E, Marcora SM. Physiological assessment of aerobic training in soccer. J Sports Sci, 2005; 23(6):586-592

Jones AM, Drust JH. A 1\% treadmill grade most accurately reflects the energetic cost of outdoor running. J Sports Sci, 1996; 14(4): 321-327

Lucia A, Hoyos J, Perez M, Santalla A, Earnest CP, Chicharro JL. Which laboratory variable is related with time trial performance time in the Tour de France? Br J Sports Med, 2004; 38(5): 636-640

McLaughlin JE, Howley ET, Bassett DR, Thompson DL, Fitzhugh EC. Test of Classic Model for Predicting Endurance Running Performance. Med Sci Sports Exerc, 2010; 42(5): 911-997

Malone S, Solan B, Collins DK, Doran DA. The positional match running performance in elite Gaelic football. J Strength Cond Res, 2015 [E-Pub Ahead of Print] Dec 18.

Manzi V, Bovenzi A, Impellizzeri FM, Carminati I, Castagna C. Individual training-load and aerobic-fitness variables in premiership soccer players during the precompetitive season. J Strength Cond Res, 2013; 27(3):631-636

McLaren SJ, Weston M, Smith A, Cramb R, Portas MD. Variability of physical performance and player match loads in professional rugby union. J Sci Med Sport 2015; [Epub Ahead of Print] Jun 5. pii: S14402440(15)00124-3, DOI: 10.1016/j.jsmas.2015.05.010

Murphy JC, Gissane C, Blake C. Injury in elite county hurling: a prospective study. Br J Sports Med, 2007; 46(2): 138-142

Paul DJ, Bradley PS, Nassis G. Factors affecting match running performance of elite soccer players: Shedding some light on the complexity. Int J Sports Physiol Perform, 2015; 10: 516-519

Reilly T, Collins K. Science and the Gaelic sports: Gaelic football and hurling. Euro J Sport Sci, 2008; 8(5): 231-240 
Stagno KM, Thatcher R, Van Someren KA. A modified TRIMP to quantify the in-season training load of team sport players. J Sports Sci, 2007; 25(6): 629-634

Stevens TG, De Ruiter CJ, Beek PJ, Savelsbergh GJ. Validity and reliability of 6-a-side small-sided game locomotor performance in assessing fitness in football players. J Sports Sci, 2016; 34(6): 527-534

Watson AW. Sports Injuries in the game of hurling: A one year perspective study. Am. J. Sports Med 1996; 24(3): 323-328.

Weaving D, Marshall P, Earle K, Nevill A, Abt G. Combining internal and external training load measure in professional rugby league. Int J Sports Physiol Perform, 2014; 9: 905-912

\section{Corresponding author:}

\section{Shane Malone}

Gaelic sport research centre , Institute of technology Tallaght, Tallaght, Dublin, Ireland.

Phone: 00353874132808

Email: shane.malone@mymail.ittdublin.ie 